\title{
Hallazgos clínicos y sociodemográficos en una cohorte de 4632 pacientes pediátricos con trastornos del lenguaje, del habla y del aprendizaje
}

\author{
Clinical and sociodemographic findings in a cohort of 4632 pediatric patients with \\ language, speech and learning disorders
}

\author{
Paulina C. Murphy-Ruiz', Emilio Arch-Tirado², María Guadalupe Leyva-Cárdenas', \\ Ana L. Adame-Calderón ${ }^{1}$, María Concepción Villarruel-Rivas ${ }^{1}$ y Ana L. Lino-González ${ }^{3 *}$ \\ ${ }^{1}$ Servicio de Patología del Lenguaje, Instituto Nacional de Rehabilitación Luis Guillermo Ibarra Ibarra; ${ }^{2}$ Centro Neurológico, Centro Médico ABC \\ Santa Fe; ${ }^{3}$ Subdirección de Investigación Clínica, División de Neurociencias, Servicio de Neurociencia Clínica, Área de Discapacidad del Lenguaje, \\ Voz y Audición, Instituto Nacional de Rehabilitación Luis Guillermo Ibarra Ibarra. Ciudad de México, México
}

\section{Resumen}

Objetivo: Describir y analizar aspectos clínicos y sociodemográficos de una cohorte de pacientes con trastornos del lenguaje, del habla y del aprendizaje. Método: Estudio retrospectivo descriptivo. Se seleccionaron expedientes de 4632 pacientes (años 2011 a 2015). Se calcularon medias, desviaciones estándar, porcentajes y frecuencias, Con la finalidad de evaluar si existía diferencia significativa entre variables se utilizó la prueba de ji al cuadrado y se realizó un análisis de probabilidad condicional entre las variables para obtener la probabilidad a priori y a posteriori. Resultados: Los trastornos del lenguaje, del habla y del aprendizaje se presentaron con mayor frecuencia en el sexo masculino, con 3358 pacientes (72.5\%). El diagnóstico más frecuente fue el trastorno del lenguaje, diagnosticado en 2924 pacientes (63.1\%). Se encontraron mayores incidencia y prevalencia de retraso del lenguaje en el sexo masculino $(p<0.05)$. Conclusiones: De todas las variables estudiadas, solo la incidencia de retraso en el desarrollo del lenguaje con respecto al sexo masculino fue significativa; de cada cuatro pacientes, tres eran de sexo masculino y uno de sexo femenino.

Palabras clave: Aprendizaje. Habla. Lenguaje. Trastorno.

\begin{abstract}
Objective: To describe and analyze clinical and sociodemographic aspects of a cohort of patients with language, speech and learning disorder. Method: Descriptive retrospective study. Patient records (2011 to 2015) were selected, making up a total of 4632. Averages, standard deviations, percentages and frequencies were calculated; in order to assess whether there was a significant difference between variables, chi squared test and a conditional probability analysis was performed between the variables to obtain a priori and a posteriori probability. Results: Disorders of language, speech and learning occurred more frequently in males, 3358 patients (72.5\%). The most frequent diagnosis was language development disorder, diagnosed in 2924 patients (63.1\%). A higher incidence and prevalence of language delay were found for the male sex $(p<0.05)$.
\end{abstract}

\footnotetext{
Correspondencia:

*Ana L. Lino-González

Calzada México Xochimilco, 289

Col. Arenal de Guadalupe

Fecha de recepción: 01-02-2020

Cir Cir. 2021;89(2):223-232

C.P.14389, Ciudad de México, México

Fecha de aceptación: 13-04-2020

Contents available at PubMed

E-mail: ana_onil@yahoo.com.mx

DOI: $10.24875 / \mathrm{CIRU} .20000060$

www.cirugiaycirujanos.com

0009-7411/C 2020 Academia Mexicana de Cirugía. Publicado por Permanyer. Este es un artículo open access bajo la licencia CC BY-NC-ND (http://creativecommons.org/licenses/by-nc-nd/4.0/).
} 
Conclusions: Of all the variables studied, only the incidence of delay in language development with respect to the male sex was significant; three out of four patients were male and 1 female.

Key words: Learning. Speech. Language. Disorder.

\section{Introducción}

Los trastornos del lenguaje, del habla y del aprendizaje (TLHyA) son dificultades que se presentan con regularidad en la población infantil de todo el mun$\mathrm{do}^{1,2}$, e implican que el niño exhiba una secuencia correcta del desarrollo, expresada a un ritmo más lento de lo esperado para su edad cronológica ${ }^{2-4}$. La American Speech Language Hearing Association (ASHA) define el trastorno del lenguaje (TL) como la dificultad para comprender o usar sistemas de símbolos verbales, no verbales o gráficos; pueden estar involucradas la forma del lenguaje o fonología (sonidos de una lengua y reglas que rigen su combinación), la morfología (estructura de las palabras y construcción de las formas de las palabras) y la sintaxis (orden y combinación de palabras para formar oraciones, y las relaciones entre los elementos dentro de una oración); también puede involucrar al contenido del lenguaje o semántica (significado de palabras y oraciones) y a la función del lenguaje en el proceso de comunicación o pragmática (sistema que combina los componentes del lenguaje antes mencionados para dar lugar a una comunicación funcional y socialmente apropiada $)^{3,5-8}$. Destaca que esta ausencia o dificultad en la producción del lenguaje no está relacionada con un bajo coeficiente intelectual, discapacidad física o sensorial, problemas neurológicos ni enfermedades mentales ${ }^{9,10}$. Por otro lado, la ASHA también considera al trastorno del habla $(\mathrm{TH})$ y lo define como la alteración que afecta la articulación de los sonidos del habla, la fluidez del habla o la voz. Un trastorno de la articulación se caracteriza por la producción atípica de los sonidos del habla; durante su producción se presentan sustituciones, omisiones, adiciones o distorsiones de los sonidos del lenguaje que pueden afectar la inteligibilidad. Por otra parte, un trastorno de fluidez es una interrupción en el flujo del habla caracterizada por un ritmo atípico, así como por repeticiones de sonidos, sílabas, palabras y frases, en ocasiones junto con exceso de tensión, actitud forzada y apoyo gestual. Finalmente, los trastornos de la voz se caracterizan por una producción anormal o una ausencia de calidad vocal, tono, volumen, resonancia o duración, considerada anormal para la edad y el sexo ${ }^{5}$.

Ambos trastornos se han asociado a factores biológicos y ambientales, entre los que destacan daño perinatal, prematuridad, bajo peso al nacimiento, complicaciones amnióticas, infecciones intrauterinas $^{11,12}$, nivel socioeconómico bajo, escaso nivel educativo de los padres ${ }^{13} y$ antecedentes familiares de retraso del lenguaje, del habla o de problemas de lectura ${ }^{14}$. Con respecto a su prevalencia, estudios realizados en diversas partes del mundo muestran que esta se modifica según la edad de la población estudiada, y varía del 1 al 27.5\% $\%^{12,15-21}$. Por otra parte, se ha encontrado que la tasa más alta se presenta en el sexo masculino, con una proporción de 1.5 niños por cada niña ${ }^{14,22}$.

El trastorno del aprendizaje (TA) es una condición caracterizada por dificultad para aprender habilidades académicas, y es posible observar habilidades educativas significativamente por debajo de lo esperado para la edad cronológica, afectando de manera importante las habilidades de lectura, escritura, matemáticas ${ }^{23}$, comprensión auditiva, expresión oral y razonamiento ${ }^{24}$. La dislexia evolutiva o dislexia del desarrollo es el trastorno del aprendizaje más frecuente $(80 \%)^{25}$ y se caracteriza por un retraso del lenguaje que afecta los componentes fonológicos, semánticos y sintácticos de la lectoescritura, observándose en estos niños una pobre comprensión lectora y dificultad en la lectura expresiva, en la redacción espontánea ${ }^{14}$ y en la ortografía ${ }^{26}$. Se reporta una prevalencia del $5-15 \%{ }^{24,27-29}$, e incluso del $3-20 \%$ en la población escolar ${ }^{26,30}$.

En un estudio realizado en Nueva York se encontró que el sexo masculino es más propenso a presentar dificultad para el aprendizaje (relación 9:6) ${ }^{28}$ y en China se ha reportado que el diagnóstico de dislexia es más frecuente en los varones (relación 2:1) ${ }^{31}$. En lo que respecta a las dificultades para las matemáticas, se refiere que en general la tasa de prevalencia es del 3-7\% en la población escolar ${ }^{23}$.

El objetivo de este artículo es describir y analizar aspectos clínicos y sociodemográficos de una cohorte de pacientes con TLHyA. 


\section{Método}

Se diseñó un estudio retrospectivo descriptivo y se analizó información extraída de la base de datos del periodo 2011-2015 del Servicio de Patología del Lenguaje (SPL) de un Instituto Nacional de Salud con sede en la Ciudad de México. La base de datos contiene información de los pacientes que asistieron o fueron referidos por presentar TLHyA, además de información sociodemográfica y clínica.

\section{Muestra}

Se estudiaron todos los ingresos presentados en un periodo de 5 años, desde el 1 de enero de 2011 hasta el 31 de diciembre de 2015, y se seleccionaron todos los pacientes de 1 a 13 años cuyo ingreso al hospital estuviera relacionado con dificultades del lenguaje, del habla y del aprendizaje.

\section{Variables}

- Variables sociodemográficas: sexo, edad, lugar de origen, lugar de residencia, nivel de instrucción del paciente, edad de la madre y escolaridad de ambos padres.

- Variables clínicas: diagnóstico, control prenatal, tipo de parto, desarrollo psicomotor, desarrollo del lenguaje y antecedentes heredofamiliares.

\section{Análisis estadístico}

Se realizó un análisis descriptivo inicial de los datos y se calcularon medias y desviaciones estándar para las variables cuantitativas, así como porcentajes y frecuencias para las variables cualitativas. Con la finalidad de evaluar si existía o no diferencia significativa entre las variables del estudio y el sexo de los pacientes se utilizó la prueba de ji al cuadrado, y para conocer la probabilidad condicional entre las variables del estudio se realizó análisis de probabilidad conjunta $P(A \cap B)=P(A) \cdot P(B)$, que es la base para obtener la probabilidad a priori $P(B \cap A i)$ y a posteriori $P(A i \cap B)$ utilizado en el teorema de Bayes.

\section{Aspectos éticos}

El protocolo fue aprobado por el Comité Institucional de Investigación y Ética. Todos los padres o tutores de los pacientes, cuando estos ingresaron al hospital, firmaron un consentimiento informado autorizando el uso de los datos con carácter anónimo.

\section{Resultados}

El número de pacientes que ingresaron al hospital por presentar TLHyA en el periodo estudiado fue de 4632. Respecto al sexo, se contabilizaron 3358 niños $(72.5 \%)$ y $1274(27.5 \%)$ niñas, con edades de 1 a 13 años (rango de 12 años) y un promedio de edad de $5.32 \pm 2$ años (media \pm desviación estándar). Con referencia al lugar de origen, 4593 (99,1\%) pacientes nacieron en 30 de las 32 entidades federativas, 35 $(0,7 \%)$ en el extranjero y $4(0.1 \%)$ en lugar desconocido. La Ciudad de México fue la principal entidad, con 3894 (84.1\%) pacientes (2820 niños y 1074 niñas), seguida del Estado de México con 489 (10.8\%) pacientes (364 niños, 125 niñas) y Puebla con 26 (0.6\%) pacientes (19 niños y 7 niñas). Con respecto al lugar de residencia, fueron 21 las entidades referidas, de las cuales las más frecuentes fueron Ciudad de México con 3974 (85.8\%) pacientes (2883 niños y 1091 niñas) y Estado de México con 580 (12.5\%) pacientes (419 niños y 161 niñas) (Tabla 1).

La edad de las madres se ubicó de 10 a 50 años (rango de 40 años) y su promedio de edad fue de 26.8 \pm 6.4 años. Respecto a la escolaridad al momento del ingreso, 2269 (49\%) pacientes (1641 niños y 628 niñas) cursaban preescolar y 1802 (38.9\%) pacientes (1311 niños y 491 niñas) se encontraban cursando la educación básica o primaria. En cuanto a escolaridad del padre, la más frecuente fue secundaria completa en 1017 (22\%) casos (744 niños y 273 niñas), seguida de bachillerato completo en 744 (16.1\%) casos (521 niños y 223 niñas). La escolaridad de la madre más frecuente fue secundaria completa en 1123 (24.2\%) casos (839 niños y 284 niñas), seguida de bachillerato completo en $821(17.7 \%)$ casos (581 niños y 240 niñas) (Tabla 2).

El diagnóstico más frecuente fue TL, presente en 2924 (63.1\%) pacientes (2124 niños y 800 niñas), seguido de TA en 1646 (35.5\%) pacientes (1186 niños y 460 niñas) y de TH en 62 (1.4\%) pacientes (48 niños y 14 niñas). Al realizar un análisis más fino de los diagnósticos recibidos se encontró que, en lo que respecta a los $\mathrm{TL}$, los más comunes fueron el trastorno fonológico sintáctico, presente en 1498 (32.3\%) pacientes (1098 niños y 400 niñas) y el trastorno de programación fonológica en 1402 (30.3\%) pacientes (1008 niños y 394 niñas); en cuanto a los TH, el más común fue la dislalia fisiológica, observada en 55 $(1.2 \%)$ pacientes (42 niños y 13 niñas); y en lo 
Tabla 1. Lugar de origen y de residencia de los pacientes con trastornos del lenguaje, del habla y del aprendizaje

\begin{tabular}{|c|c|c|c|c|c|c|c|c|}
\hline & \multicolumn{2}{|c|}{ Origen } & \multicolumn{2}{|c|}{ Sexo } & \multicolumn{2}{|c|}{ Residencia } & \multicolumn{2}{|c|}{ Sexo } \\
\hline & $\mathrm{n}$ & $\%$ & Niño & Niña & $\mathrm{n}$ & $\%$ & Niño & Niña \\
\hline Aguascalientes & 22 & 0.5 & 13 & 9 & 2 & 0 & 1 & 1 \\
\hline Baja California & 4 & 0.1 & 1 & 3 & 1 & 0 & 0 & 1 \\
\hline Baja California Sur & 6 & 0.1 & 2 & 4 & - & - & - & - \\
\hline Campeche & 1 & 0 & 0 & 1 & 3 & 0.1 & 3 & 0 \\
\hline Coahuila & 1 & 0 & 1 & 0 & - & - & - & - \\
\hline Chiapas & 5 & 0.1 & 4 & 1 & 2 & 0 & 1 & 1 \\
\hline Chihuahua & 4 & 0.1 & 3 & 1 & - & - & - & - \\
\hline Ciudad de México & 3894 & 84.1 & 2820 & 1074 & 3974 & 85.8 & 2883 & 1091 \\
\hline Durango & 6 & 0.1 & 3 & 3 & - & - & - & - \\
\hline Estado de México & 489 & 10.6 & 364 & 125 & 580 & 12.5 & 419 & 161 \\
\hline Guanajuato & 8 & 0.2 & 7 & 1 & - & - & - & - \\
\hline Guerrero & 19 & 0.4 & 14 & 5 & 13 & 0.3 & 8 & 5 \\
\hline Hidalgo & 12 & 0.3 & 9 & 3 & 8 & 0.2 & 6 & 2 \\
\hline Jalisco & 7 & 0.2 & 5 & 2 & 5 & 0.1 & 5 & 0 \\
\hline Michoacán & 10 & 0.2 & 7 & 3 & 5 & 0.1 & 4 & 1 \\
\hline Morelos & 13 & 0.3 & 10 & 3 & 9 & 0.2 & 6 & 3 \\
\hline Nayarit & 1 & 0 & 1 & 0 & - & - & - & - \\
\hline Nuevo León & 4 & 0.1 & 3 & 1 & - & - & - & - \\
\hline Oaxaca & 8 & 0.2 & 6 & 2 & - & - & - & - \\
\hline Puebla & 26 & 0.6 & 19 & 7 & 5 & 0.1 & 4 & 1 \\
\hline Querétaro & 7 & 0.2 & 6 & 1 & 3 & 0.1 & 2 & 1 \\
\hline Quintana Roo & 5 & 0.1 & 4 & 1 & 2 & 0 & 2 & 0 \\
\hline San Luis Potosí & 3 & 0.1 & 2 & 1 & 1 & 0 & 0 & 1 \\
\hline Sinaloa & 3 & 0.1 & 3 & 0 & 2 & 0 & 1 & 1 \\
\hline Sonora & 3 & 0.1 & 0 & 3 & 1 & 0 & 0 & 1 \\
\hline Tabasco & 1 & 0 & 1 & 0 & - & - & - & - \\
\hline Tamaulipas & 5 & 0.1 & 1 & 4 & - & - & - & - \\
\hline Tlaxcala & 6 & 0.1 & 4 & 2 & 5 & 0.1 & 4 & 1 \\
\hline Veracruz & 17 & 0.4 & 12 & 5 & 10 & 0.2 & 8 & 2 \\
\hline Zacatecas & 3 & 0.1 & 3 & 0 & 1 & 0 & 1 & 0 \\
\hline Extranjero & 35 & 0.8 & 28 & 7 & - & - & - & - \\
\hline Desconocido & 4 & 0.1 & 2 & 2 & - & - & - & - \\
\hline
\end{tabular}

referente a los TA, el diagnóstico más frecuente fue dislexia mixta, encontrada en $916(19.8 \%)$ pacientes (664 niños y 252 niñas) (Tabla 3).
Con respecto a los antecedentes, se encontró que 4510 (97.4\%) madres (de 3273 niños y 1237 niñas) refirieron haber tenido control prenatal y 2409 (52\%) 
Tabla 2. Escolaridad de pacientes y progenitores

\begin{tabular}{|c|c|c|c|c|c|}
\hline & $\mathrm{n}$ & $\%$ & Niños & Niñas & $\chi^{2}$ \\
\hline \multicolumn{6}{|c|}{ Nivel de instrucción del paciente } \\
\hline Ninguna & 502 & 10.8 & 359 & 143 & NS \\
\hline Preescolar & 2269 & 49 & 1641 & 628 & \\
\hline Primaria incompleta & 1802 & 38.9 & 1311 & 491 & \\
\hline Primaria completa & 7 & 0.2 & 6 & 1 & \\
\hline Secundaria & 6 & 0.1 & 3 & 3 & \\
\hline Educación especial & 4 & 0.1 & 3 & 1 & \\
\hline Guardería & 42 & 0.9 & 35 & 7 & \\
\hline \multicolumn{6}{|c|}{ Nivel de instrucción de la madre } \\
\hline Analfabeta & 41 & 0.9 & 24 & 17 & NS \\
\hline Sabe leer y escribir & 35 & 0.8 & 21 & 14 & \\
\hline Primaria incompleta & 95 & 2.1 & 74 & 21 & \\
\hline Primaria completa & 311 & 6.7 & 215 & 96 & \\
\hline Secundaria incompleta & 203 & 4.4 & 149 & 54 & \\
\hline Secundaria completa & 1123 & 24.2 & 839 & 284 & \\
\hline Bachillerato incompleto & 399 & 8.6 & 294 & 105 & \\
\hline Bachillerato completo & 821 & 17.7 & 581 & 240 & \\
\hline Carrera técnica & 397 & 8.6 & 291 & 106 & \\
\hline Licenciatura incompleta & 208 & 4.5 & 139 & 69 & \\
\hline Licenciatura completa & 502 & 10.8 & 372 & 130 & \\
\hline Posgrado & 40 & 0.9 & 29 & 11 & \\
\hline Se desconoce & 457 & 9.9 & 330 & 127 & \\
\hline \multicolumn{5}{|c|}{ Nivel de instrucción del padre } & NS \\
\hline Analfabeto & 28 & 0.6 & 19 & 9 & \\
\hline Sabe leer y escribir & 29 & 0.6 & 18 & 11 & \\
\hline Primaria incompleta & 79 & 1.7 & 57 & 22 & \\
\hline Primaria completa & 291 & 6.3 & 209 & 82 & \\
\hline Secundaria incompleta & 177 & 3.8 & 136 & 41 & \\
\hline Secundaria completa & 1017 & 22 & 744 & 273 & \\
\hline Bachillerato incompleto & 307 & 6.6 & 243 & 64 & \\
\hline Bachillerato completo & 744 & 16.1 & 521 & 223 & \\
\hline Carrera técnica & 206 & 4.4 & 144 & 62 & \\
\hline Licenciatura incompleta & 163 & 3.5 & 110 & 53 & \\
\hline Licenciatura completa & 507 & 10.9 & 375 & 132 & \\
\hline Posgrado & 33 & 0.7 & 23 & 10 & \\
\hline Se desconoce & 1051 & 22.7 & 759 & 292 & NS \\
\hline
\end{tabular}

madres (de 1741 niños y 668 niñas) informaron parto por cesárea. En cuanto al desarrollo psicomotor de los pacientes, se refirió normal en $3717(80.2 \%)$ casos (2719 niños y 998 niñas); el desarrollo del lenguaje se notificó con retraso en 3661 (79\%) casos (2682 niños y 979 niñas). Al calcular la ji al cuadrado se obtuvo un valor de $p=0.05$, lo que significa que existe mayor incidencia de problemas del lenguaje en el sexo masculino (lo cual coincide con lo reportado en la literatura y se destaca que fue la única variable que mostró diferencia significativa con respecto al sexo de los pacientes). En lo referente a antecedentes heredofamiliares, en $2220(47.9 \%)$ casos (1601 niños y 619 niñas) se informó trastorno de comunicación (lenguaje, habla o aprendizaje) (Tabla 4).

Para obtener la probabilidad conjunta entre los TLHyA con respecto al sexo de los pacientes se calculó la intersección probabilística de las proporciones $P(A \cap B)=P(A) \cdot P(B)$, en donde $A$ y $B$ fueron calculadas con la proporción a analizar, es decir, el total de pacientes de sexo masculino o femenino entre el total de la muestra, y el total de pacientes de sexo masculino o femenino en cada uno de los trastornos entre el total de pacientes por sexo. De esta manera, para los cálculos la proporción de pacientes de sexo masculino fue $\frac{3358}{4632}=0.7249(72.49 \%)$ y la proporción de pacientes de sexo femenino fue $\frac{1274}{4632}=0.275$ (27.5\%), de modo que la probabilidad conjunta con respecto al sexo de los pacientes y los TL fue: $(A)$ pacientes de sexo masculino entre el total de la muestra y $(B)$ pacientes de sexo masculino con TL entre el total de pacientes de sexo masculino, es decir, $P(A \cap B)=P\left(\frac{3358}{4632} \cap \frac{2124}{3358}\right)=0.4584$; esto es, la probabilidad de que un paciente sea de sexo masculino y presente TL es del $45.84 \%$. Por otra parte, considerando $(A)$ pacientes de sexo femenino entre el total de la muestra y pacientes $(B)$ de sexo femenino con TL entre el total de pacientes de sexo femenino, es decir, $P(A \cap B)=P\left(\frac{1274}{4632} \cap \frac{800}{1274}\right)=0.1726$, significa que, del total de la muestra, la probabilidad de que un paciente sea de sexo femenino y presente TL es del $17.26 \%$. Ahora bien, en lo referente al cálculo de la probabilidad condicional a posteriori, $P(B \cap A i)$, considerando las variables $T L$ y sexo de los pacientes, utilizando el teorema de Bayes, se obtuvo que la probabilidad condicional a posteriori de que un paciente llegue al SPL, sea diagnosticado con TL y sea de sexo masculino es de 0.7264 (72.64\%), mientras que la probabilidad condicional a posteriori de que un paciente llegue al SPL, sea diagnosticado con TL y sea del sexo femenino es de 0.2735 (27.35\%) (Tabla 5).

\section{Discusión}

El presente estudio aporta información que permite conocer las características de los pacientes diagnosticados con TLHyA de una cohorte de pacientes pediátricos atendidos en un Instituto Nacional de Salud en México. Al tratarse de un hospital de referencia y especializado, es elevado el número de pacientes (4632) en edad pediátrica que ingresaron. Analizando esta información con respecto a cifras nacionales oficiales, encontramos que el Instituto 
Tabla 3. Diagnóstico

\begin{tabular}{|c|c|c|c|c|c|}
\hline & $\mathbf{n}$ & $\%$ & Niños & Niñas & $\chi^{2}$ \\
\hline Trastornos & & & & & NS \\
\hline Lenguaje & 2924 & 63.1 & 2124 & 800 & \\
\hline Habla & 62 & 1.4 & 48 & 14 & \\
\hline Aprendizaje & 1646 & 35.5 & 1186 & 460 & \\
\hline \multicolumn{6}{|l|}{ Diagnóstico específico } \\
\hline Lenguaje & & & & & NS \\
\hline Afasia infantil & 4 & 0.1 & 2 & 2 & \\
\hline Dispraxia verbal & 14 & 0.3 & 12 & 2 & \\
\hline Trastorno fonológico sintáctico & 1498 & 32.3 & 1098 & 400 & \\
\hline Trastorno de programación fonológica & 1402 & 30.3 & 1008 & 394 & \\
\hline Trastorno semántico pragmático & 6 & 0.1 & 4 & 2 & \\
\hline Habla & & & & & NS \\
\hline Disglosia & 1 & 0 & 1 & 0 & \\
\hline Dislalia fisiológica & 55 & 1.2 & 42 & 13 & \\
\hline Rotacismo & 3 & 0.1 & 3 & 0 & \\
\hline Disartria & 3 & 0.1 & 2 & 1 & \\
\hline Aprendizaje & & & & & NS \\
\hline Dislexia diseidética & 289 & 6.2 & 211 & 78 & \\
\hline Dislexia disfonética & 440 & 9.5 & 310 & 130 & \\
\hline Dislexia mixta & 916 & 19.8 & 664 & 252 & \\
\hline Discalculia & 1 & 0 & 1 & 0 & \\
\hline
\end{tabular}

Nacional de Estadística y Geografía (INEGI), para el año 2014, refiere que 7.1 millones de habitantes (6\%) presenta algún tipo de discapacidad, entre las que considera la dificultad para aprender (38.8\%) y la dificultad para hablar o comunicarse $(18 \%)^{32}$. Con respecto al sexo de los pacientes, nuestros datos concuerdan con lo reportado en estudios internacionales en los que se establece que el sexo masculino es un factor de riesgo ${ }^{13,14,22,28,31,33}$. Por otro lado, analizando la edad de los pacientes, estos se ubicaron entre 1 y 13 años, lo cual concuerda con los datos del INEGI, que destacan que las dificultades para aprender, hablar o comunicarse se encuentran con mayor frecuencia y gravedad en la población menor de 15 años $^{32}$. Continuando con este tema, vale la pena destacar que la Organización Mundial de la Salud considera que los tres primeros años de vida son un periodo crítico, el cual se caracteriza por un desarrollo acelerado del sistema nervioso que favorece el crecimiento y el desarrollo integral del niño $0^{4}$ También se ha sugerido que los trastornos relacionados con la expresión del lenguaje pueden ser notados por los padres entre los 18 meses y los 3 años de edad; sin embargo, es frecuente escuchar que el niño se encuentra en proceso de desarrollo o de adquisición del lenguaje y que cuenta con tiempo suficiente para desarrollarlo de forma adecuada, por lo que muchas veces no se le da importancia y es derivado con el especialista de forma tardía ${ }^{14}$.

El INEGI también hace referencia a que, en México, aproximadamente la mitad de la población con discapacidad (49.6\%) se concentra en siete entidades federativas, entre las que destacan la Ciudad de México $(5.8 \%)$ y el Estado de México $(14.6 \%)^{32}$. En nuestro estudio, estas dos entidades concentran el mayor número de pacientes, y en este sentido vale la pena considerar que el Instituto donde se realizó este estudio se encuentra ubicado en la Ciudad de México, razón suficiente para que la mayor población que acude a solicitar atención se encuentre viviendo en esta ciudad, o bien en el Estado de México, que al ser parte del área conurbada cuenta con los medios de transporte que facilitan el traslado de los pacientes no solo durante la detección del problema, sino también para el tratamiento y el seguimiento médico especializado.

Encontramos que las madres más jóvenes tenían 10 años de edad y las más añosas 50 años; el promedio de edad y la desviación estándar $(26.8 \pm 6.4$ años) se ubicaron dentro de los parámetros de edad más recomendados para ejercer la maternidad. Consideramos la edad de las madres, ya que suelen ser las que habitualmente se hacen cargo de brindar atención y cuidado a los pacientes, y en este sentido destacamos que en la revisión realizada se encontró 
Tabla 4. Antecedentes

\begin{tabular}{|c|c|c|c|c|c|c|}
\hline & $n$ & $\%$ & Niños & Niñas & $\chi^{2}$ & $p$ \\
\hline $\begin{array}{l}\text { Control prenatal } \\
\text { Sí } \\
\text { No } \\
\text { Se desconoce }\end{array}$ & $\begin{array}{c}4510 \\
101 \\
21\end{array}$ & $\begin{array}{c}97.4 \\
2.2 \\
0.5\end{array}$ & $\begin{array}{c}3273 \\
73 \\
12\end{array}$ & $\begin{array}{c}1237 \\
28 \\
9\end{array}$ & NS & \\
\hline $\begin{array}{l}\text { Tipo de parto } \\
\text { Eutócico } \\
\text { Cesárea } \\
\text { Distócico } \\
\text { Fortuito } \\
\text { Se desconoce }\end{array}$ & $\begin{array}{c}2049 \\
2409 \\
74 \\
79 \\
21\end{array}$ & $\begin{array}{c}44.2 \\
52 \\
1.6 \\
1.7 \\
0.5\end{array}$ & $\begin{array}{c}1485 \\
1741 \\
58 \\
62 \\
12\end{array}$ & $\begin{array}{c}564 \\
668 \\
16 \\
17 \\
9\end{array}$ & & \\
\hline $\begin{array}{l}\text { Desarrollo psicomotor } \\
\text { Normal } \\
\text { Con retraso } \\
\text { Se desconoce }\end{array}$ & $\begin{array}{c}3717 \\
884 \\
31\end{array}$ & $\begin{array}{c}80.2 \\
19.1 \\
0.7\end{array}$ & $\begin{array}{c}2719 \\
619 \\
20\end{array}$ & $\begin{array}{c}998 \\
265 \\
11\end{array}$ & NS & \\
\hline $\begin{array}{l}\text { Desarrollo del lenguaje } \\
\text { Normal } \\
\text { Con retraso } \\
\text { Se desconoce }\end{array}$ & $\begin{array}{c}934 \\
3661 \\
37\end{array}$ & $\begin{array}{c}20.2 \\
79 \\
0.8\end{array}$ & $\begin{array}{c}650 \\
2682 \\
26\end{array}$ & $\begin{array}{c}284 \\
979 \\
11\end{array}$ & 5.0161 & $<0.05$ \\
\hline $\begin{array}{l}\text { Antecedentes heredofamiliares } \\
\text { Ninguno } \\
\text { Trastorno de comunicación } \\
\text { Trastorno psiquiátrico } \\
\text { Trastorno neurológico } \\
\text { Trastorno congénito } \\
\text { Se desconoce } \\
\text { Trastorno genético } \\
\text { Trastorno de comunicación y neurológico } \\
\text { Trastornos psiquiátrico y neurológico } \\
\text { Trastornos de comunicación y psiquiátrico } \\
\text { Trastorno de comunicación y congénito } \\
\text { Trastorno de comunicación y genético }\end{array}$ & $\begin{array}{c}1657 \\
2220 \\
92 \\
142 \\
44 \\
12 \\
106 \\
118 \\
20 \\
125 \\
85 \\
11\end{array}$ & $\begin{array}{l}35.8 \\
47.9 \\
2 \\
3.1 \\
0.9 \\
0.3 \\
2.3 \\
2.5 \\
0.4 \\
2.7 \\
1.8 \\
0.2\end{array}$ & $\begin{array}{c}1211 \\
1601 \\
61 \\
105 \\
29 \\
5 \\
77 \\
87 \\
19 \\
95 \\
61 \\
7\end{array}$ & $\begin{array}{c}446 \\
619 \\
31 \\
37 \\
15 \\
7 \\
29 \\
31 \\
1 \\
30 \\
24 \\
4\end{array}$ & NS & \\
\hline
\end{tabular}

NS: no significativo.

que se ha relacionado la edad materna avanzada como un factor de riesgo para el desarrollo de trastornos de la comunicación ${ }^{33}$.

En lo referente a la escolaridad de los pacientes, el $49 \%$ se ubicó en el primer nivel de educación básica o preescolar, y el $38.9 \%$ en el segundo nivel o primaria. El rango de edad habitual aproximado para cursar el preescolar de acuerdo con el Sistema Educativo Nacional es de 3 a 5 años $^{34}$, y podemos suponer que los pacientes que acudieron al Instituto en este rango de edad lo hicieron por sospecha de TL o TH. En lo que respecta a la educación primaria, en este nivel de estudios se incorporan niños de 6 a 12 años $^{34}$, y por lo tanto es posible que en este rango estén ubicados pacientes con detección tardía de TL o TH, sumándose también aquellos pacientes que contaban con alguno de estos diagnósticos y que evolucionaron a un TA o presentaron como tal un TA. Algunos autores estipulan que los niños con TL tienen un riesgo cuatro o cinco veces mayor de presentar dificultades para la lectura y para desarrollar dificultades en las habilidades sociales, además de problemas en escritura, ortografía, matemáticas y atención, y presencia de dislexia del desarrollo y niveles más bajos de rendimiento escolar ${ }^{2,3,6,12,13,35}$.

Nos interesó conocer la escolaridad de ambos padres y encontramos que es similar; la mayoría refirió tener estudios de secundaria completa, es decir, concluyeron la tercera y última etapa de la educación básica. Algunos autores refieren que el nivel de estudios de ambos progenitores afecta el desarrollo del lenguaje y del aprendizaje de los niños ${ }^{35,36}$; otros consideran que el nivel educativo de la madre es fundamental y sugieren que las madres analfabetas o con bajo nivel de estudios representan un factor de riesgo para el desarrollo de $\mathrm{TL}$ y de $T A^{31,37-39}$, y que aquellas con estudios de nivel superior representan un factor protector ${ }^{33}$.

Con respecto al desarrollo psicomotor, en nuestro estudio el $80.2 \%$ de los padres refirió que el desarrollo de los pacientes había sido normal; sin embargo, valdría la pena realizar una evaluación objetiva por algún 
Tabla 5. Probabilidad a priori y a posteriori con respecto al sexo del paciente y el trastorno diagnosticado

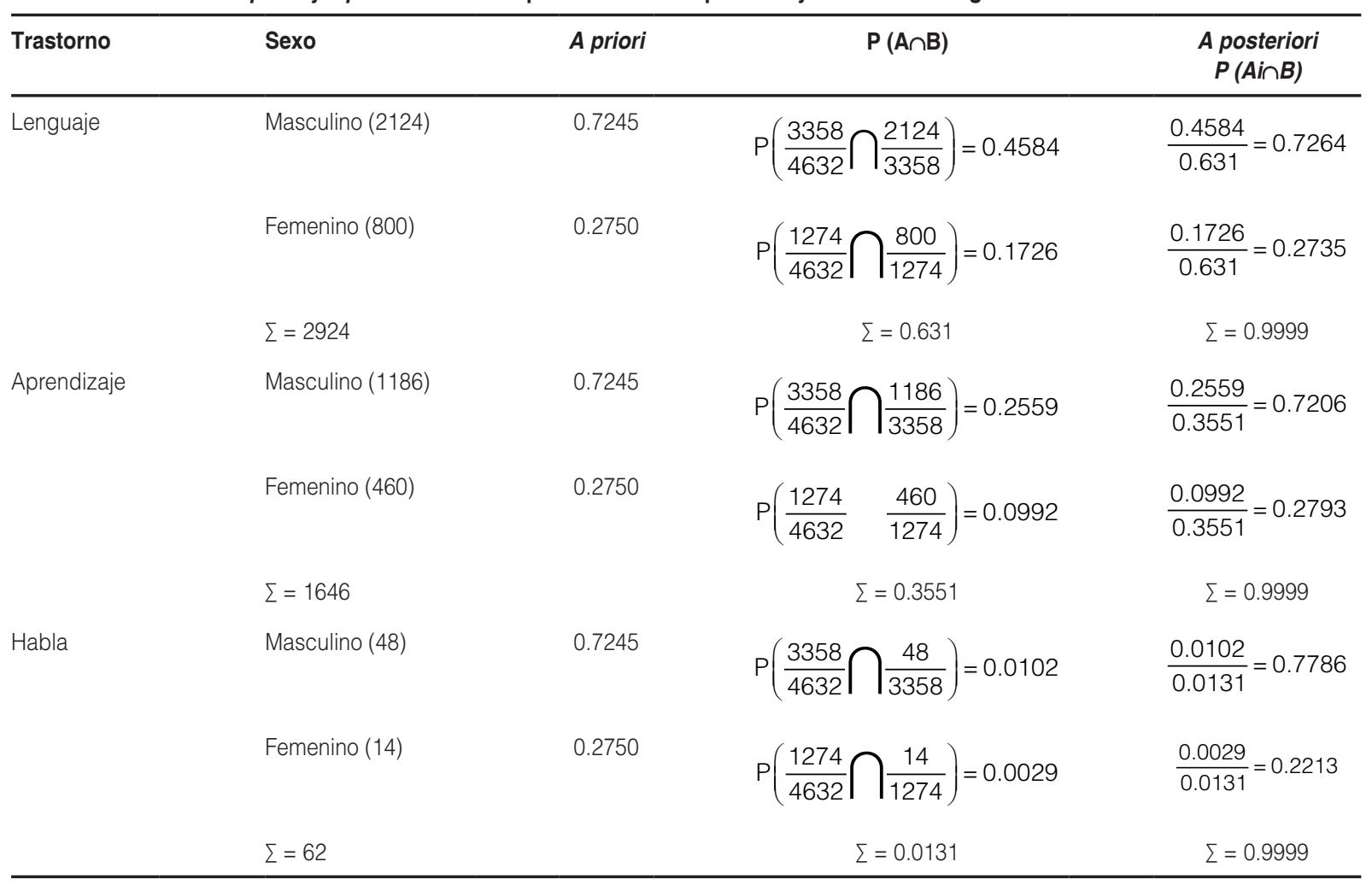

especialista psicomotricista que permita verificar esta información, ya que son varios los investigadores que han encontrado una relación entre los trastornos motores y el desarrollo de TLHyA $2,9,40$. Okuda y Pinheiro ${ }^{41}$ consideran que cuando el niño es capaz de lograr un buen control motor se facilita la construcción de los conceptos básicos para el desarrollo intelectual, constituyendo una relación directa entre el desarrollo cognitivo y el desarrollo motor. Un deficiente rendimiento motor es evidente cuando el niño presenta torpeza, falta de equilibrio, retraso en los hitos del desarrollo (gatear, caminar, sentarse), o pobre desempeño en actividades como lanzar, atrapar, correr, patear, saltar, recortar, colorear y escribir; todos ellos son considerados aspectos que interferirán en el rendimiento académico.

En cuanto a los antecedentes heredofamiliares, se encontró que el $47.9 \%$ de los pacientes tenía o había tenido familiares con TLHyA, dato que es concordante con lo reportado en la literatura, ya que para los trastornos que nos ocupan se refiere una afectación significativa por predisposición genética ${ }^{12,33,40}$. Los niños con un historial familiar de habla poco inteligible, disfemia, retraso en la adquisición del lenguaje y vocabulario limitado tienen cuatro veces más probabilidad de presentar $\mathrm{TH} \circ \mathrm{TL}^{13,39}$. Así mismo, se ha demostrado que la prevalencia de dislexia del desarrollo es alta en los descendientes de padres con dificultades en la lectura ${ }^{35}$; aunado a esto, existe evidencia de que la dislexia del desarrollo se asocia a múltiples genes ${ }^{25,26}$.

Considerando los resultados obtenidos al calcular la probabilidad conjunta, destaca la elevada probabilidad de que un paciente presente TL, siendo para el sexo masculino del $45.8 \%$ y para el femenino del $17.2 \%$, en comparación con los pacientes con TA o TH. Con respecto a la probabilidad condicional a posteriori, para el TL y el TA, considerando que en ambos sexos la proporción es prácticamente la misma, la probabilidad de que un paciente llegue al SPL, sea diagnosticado con TL o TA, y sea de sexo masculino, es del $72 \%$, mientras que la probabilidad de que llegue al SPL, sea diagnosticado con TL o TA, y sea de sexo femenino, es del $27 \%$. Llama la atención que, en lo que respecta al $\mathrm{TH}$, los resultados muestran la misma tendencia en cuanto a la proporción y se observa una mayor diferencia entre el sexo masculino y el sexo femenino; en este caso particular, la probabilidad de que un paciente llegue al SPL, sea diagnosticado con TH y sea de sexo masculino es del $77 \%$, un $5 \%$ mayor que para 
los pacientes con TL y TA; y la probabilidad de que un paciente llegue al SPL, sea diagnosticado con $\mathrm{TH}$ y sea de sexo femenino es del $22 \%$, un $5 \%$ menor en comparación con las pacientes con TL y TA.

Un aspecto que merece la pena destacar tiene que ver con el tratamiento que requieren estos pacientes una vez que se ha detectado el problema y se ha realizado el diagnóstico; lo ideal es que esta intervención sea temprana y por especialistas calificados, pero esto se puede complicar, por ejemplo, en los pacientes que presentan trastornos leves, motivo por el cual se dificulta la tipificación del problema, lo que conduce a una identificación y un diagnóstico tardíos. En el caso de los trastornos graves, estos pueden volverse persistentes y continuar durante la edad adulta ${ }^{2,40,42,43}$. Se ha podido establecer, en diferentes estudios, que los adultos que fueron diagnosticados con TL y TH en la infancia y que no fueron tratados, o recibieron una intervención tardía, tienen más probabilidad de estar desempleados, tener menores ingresos, desempeñarse en trabajos menos calificados o tener pocas posibilidades de acceso a educación o capacitación $n^{3,12,27,42}$.

Otro aspecto importante está relacionado con lo que se reporta sobre la intervención, el seguimiento médico o terapéutico, y el desarrollo socioemocional de estos niños, el cual se refiere con importantes dificultades $^{6,44,45}$. Por ejemplo, se ha reportado que los TA se asocian con altas tasas de deserción de la escuela secundaria, bajo rendimiento académico, falta de oportunidades para progresar en el sistema educativo o de capacitación, altos niveles de angustia ${ }^{27,42,}$, ansiedad social, episodio maníaco, trastorno afectivo bipolar y depresión psicótica ${ }^{2,42}$. Los adolescentes con un TL no resuelto muestran dificultades sociales ${ }^{1,42}$ y niveles elevados de agresión, lo cual podría explicarse por la presencia de un alto grado de frustración e incapacidad para emplear las habilidades lingüísticas como puente o facilitador en la interacción con otros y para la resolución efectiva de problemas ${ }^{43}$. Es importante destacar que la capacidad lingüística no solo cumple con la función de la comunicación, sino que también es un recurso de autorregulación cognitiva y conductual, ya que facilita el conocimiento y la comprensión de las emociones ${ }^{44}$. Se ha sugerido que una intervención temprana y adecuada durante la niñez puede interrumpir el riesgo para desarrollar dificultades socioemocionales y del comportamiento, relacionadas muchas veces con el uso de sustancias nocivas y el incumplimiento de reglas sociales ${ }^{43}$.

Algunos autores sugieren que, además del tratamiento terapéutico especializado por parte de terapistas del lenguaje y del habla (logopedas, patólogos del lenguaje y del habla, fonoaudiólogos, terapeutas en comunicación humana, etc.), es importante que estos niños acudan a sesiones regulares de psicoterapia que les ayude a tener confianza en sí mismos, a facilitar su adaptación en ambientes escolares y a mejorar su calidad de vida social ${ }^{7}$.

\section{Conclusiones}

De todas las variables estudiadas, solo la incidencia de retraso en el desarrollo del lenguaje en el sexo masculino fue significativa: de cada cuatro pacientes, tres eran de sexo masculino y uno de sexo femenino.

Con respecto a la población atendida, la probabilidad condicional a priori de que los pacientes lleguen al SPL, sean diagnosticados con cualquiera de los tres trastornos y todos sean de sexo masculino, o todos sean de sexo femenino, es la misma. Por otro lado, la probabilidad condicional a posteriori muestra una importante similitud con respecto a la proporción en que se presentan los tres trastornos; sin embargo, la proporción más alta se encontró en los pacientes de sexo masculino con TH y la más baja en los de sexo femenino.

Los TLHyA representan un problema muy común en la primera infancia. Gracias a diversos estudios se ha podido establecer que se presentan en todo el mundo y que además pueden prolongarse y complicarse en la adolescencia y en la edad adulta, afectando de manera significativa el desarrollo emocional, académico, social y laboral; por lo tanto, es fundamental destacar la importancia de la detección y la intervención terapéutica temprana a cargo de médicos especialistas y de logopedas o terapeutas del lenguaje, del habla y del aprendizaje.

La implementación de programas creados para informar y orientar a padres de familia, otros familiares, profesores y médicos permitirá resolver estos problemas a edades tempranas e impedir la evolución a condiciones más graves.

\section{Conflicto de intereses}

Los autores declaran que no existe conflicto de intereses.

\section{Responsabilidades éticas}

Protección de personas y animales. Los autores declaran que los procedimientos seguidos se conformaron a las normas éticas del comité de experimentación 
humana responsable y de acuerdo con la Asociación Médica Mundial y la Declaración de Helsinki.

Confidencialidad de los datos. Los autores declaran que han seguido los protocolos de su centro de trabajo sobre la publicación de datos de pacientes.

Derecho a la privacidad y consentimiento informado. Los autores han obtenido el consentimiento informado de los pacientes y/o sujetos referidos en el artículo. Este documento obra en poder del autor de correspondencia.

\section{Bibliografía}

1. Assous A, Borghini A, Levi-Rueff M, Rittori G, Rousselot-Pailley B, Gosme $C$, et al. Children with mixed developmental language disorder have more insecure patterns of attachment. BMC Psychol. 2018;6:54.

2. Perna R, Loughan AR. Early developmental delays: a cross validation study. J Psychol Abnorm Child. 2013:2:1-5.

3. Wallace IF, Berkman ND, Watson LR, Coyne-Beasley T, Wood CT, Cullen $\mathrm{K}$, et al. Screening for speech and language delay in children 5 years old and younger: a systematic review. Pediatrics. 2015;136:e448-62.

4. World Health Organization, UNICEF. Early childhood development and disability: discussion paper. 2012. (Consultado el 25 de julio de 2019.) Disponible en: https://apps.who.int/iris/bitstream/handle/10665/75355/9789241504065 eng.pdf?sequence=1\&isAllowed=y

5. American Speech Language Hearing Association. Definitions of communication disorders and variations. ASHA. 1993;35(3 Suppl 10):40-1.

6. Weismer SE. Developmental language disorders: challenges and implications of cross-group comparisons. Folia Phoniatr Logo. 2013;65:68-77.

7. Schachinger-Lorentzon U, Kadesjö B, Gillberg C, Miniscalco C. Children screening positive for language delay at 2.5 years: language disorde and developmental profiles. 口Neuropsychiatr Dis Treat. 2018;14:3267-77.

8. Ramus F, Marshall CR, Rosen S, van der Lely HK. Phonological deficits in specific language impairment and developmental dyslexia: towards a multidimensional model. Brain. 2013;136:630-45.

9. Tomas $\mathrm{E}$, Vissers $\mathrm{C}$. Behind the scenes of developmental language disorder: time to call neuropsychology back on stage. Front Hum Neurosci. 2019;12:217.

10. Hubert-Dibon G, Bru M, Le Guen CG, Launay E, Roy A. Health-related quality of life for children and adolescents with specific language impairment: a cohort study by a learning disabilities reference center. PLoS One. 2016;11:e0166541.

11. Arias JS, Martínez JI, Tolosa A, Revuelta JC, Llacer B, Jover M. Factores genéticos en el desarrollo del lenguaje. Rev Neurol. 2010;50:101-6.

12. Al-Fadhli KY, Al-Bunaian NAR. Prevalence and social influences of delayed language development in preschool-age Saudi children. Int J Sc Res. 2017:6:1712-20.

13. Mondal N, Bhat BV, Plakkal N, Thulasingam M, Ajayan P, Poorna DR. Prevalence and risk factors of speech and language delay in children less than three years of age. J Compr Ped. 2016;7:e33173.

14. Moreno-Flagge N. Trastornos del lenguaje. Diagnóstico y tratamiento. Rev Neurol. 2013;57(Supl 1):S85-94

15. Law J, Boyle J, Harris F, Harkness A, Nye C. Prevalence and natural history of primary speech and language delay: findings from a systematic review of the literature. Int J Lang Commun Disord. 2000;35:165-88.

16. Beitchman JH, Nair R, Clegg M, Patel PG. Prevalence of speech and language disorders in 5-year-old kindergarten children in the Ottawa-Carleton region. J Speech Hear Disorders. 1986;51:98-110.

17. De Andrade CR. Speech-language idiopathic disorder prevalence in children from one to eleven years of age. Rev Saude Publica. 1997:31:495.

18. Keating D, Turrell G, Ozanne A. Childhood speech disorders: reported prevalence, comorbidity and socioeconomic profile. J Paediatr Child Health. 2001;37:431-6.

19. Tomblin JB, Records NL, Buckwalter $P$, Zhang X, Smith E, O'Brien M Prevalence of specific language impairment in kindergarten children. $\square \mathrm{J}$ Speech Lang Hear Res. 1997; 40:1245-60.

20. Norbury CF, Gooch D, Wray C, Baird G, Charman T, Simonoff E, et al. The impact of nonverbal ability on prevalence and clinical presentation of language disorder: evidence from a population study. J Child Psychol Psychiatry. 2016;57:1247-57.
21. García-Mateos M, Cinca MAM, de Santiago Herrero J, de Pedro BZ. Prevalencia de las patologías del habla, del lenguaje y de la comunicación. Un estudio a lo largo del ciclo vital. Rev Logop Foniatr Audiol. 2014;4:163-70.

22. Pinborough-Zimmerman J, Satterfield R, Miller J, Bilder D, Hossain S, McMahon W. Communication disorders: prevalence and comorbid intellectual disability, autism, and emotional/behavioral disorders. Am J Speech Lang Pathol. 2007;16:359-67.

23. Moll K, Kunze S, Neuhoff N, Bruder J, Schulte-Körne G. Specific learning disorder: prevalence and gender differences. PLoS One. 2014:9:e103537.

24. Altay MA, Görker I. Assessment of psychiatric comorbidity and WISC-R profiles in cases diagnosed with specific learning disorder according to DSM-5 criteria. Arch Neuropsychiatry. 2018;55:127-34.

25. Málaga Diéguez I. Los trastornos del aprendizaje. Definición de los distintos tipos y sus bases neurobiológicas. Bol Pediatr. 2010;50:43-7.

26. Liu L, Wang J, Shao S, Luo X, Kong $R$, Zhang X et al. Descriptive epidemiology of prenatal and perinatal risk factors in a Chinese population with reading disorder. Sci Rep. 2016;6:36697.

27. Peters L, Bulthé J, Daniels N, De Beeck HO, De Smedt B. Dyscalculia and dyslexia: different behavioral, yet similar brain activity profiles during arithmetic. Neuroimage Clin. 2018;18:663-74.

28. New York City Department of Health and Mental Hygiene. Developmental conditions and delays in New York City children. 2011. (Consultado el 16 de agosto de 2019.) Disponible en: https://www1.nyc.gov/assets/ doh/downloads/pdf/epi/databrief8.pdf

29. Lorenzo ST. La dislexia y las dificultades en la adquisición de la lectoescritura. Profesorado. Revista de Currículum y Formación de Profesorado. 2017;21:423-32.

30. Adlof SM, Hogan TP. Understanding dyslexia in the context of developmental language disorders. Lang Speech Hear Ser. 2018:49:762-73.

31. Zhao H, Zhang B, Chen Y, Zhou X, Zuo P. Environmental risk factors in Han and Uyghur children with dyslexia: a comparative study. PLoS One. 2016;11:e0159042.

32. Instituto Nacional de Estadística y Geografía. La discapacidad en México, datos del 2014. Versión 2017. (Consultado el 15 de agosto de 2019.) Disponible en: http://conadis-transparencia.org/transparencia focalizada/La discapacidad en Mexico datos_al_2014 Version_2017.pdf.

33. Moriano-Gutiérrez A, Colomer-Revuelta J, San Juan J, Carot-Sierra JM. Variables ambientales y genéticas relacionadas con alteraciones en la adquisición del lenguaje en la infancia. Rev Neurol. 2017;64:31-7.

34. Narro Robles J, Martuscelli Quintana J, Barzana García E. Plan de diez años para desarrollar el Sistema Educativo Nacional. 2012. (Consultado el 26 de agosto de 2018.) Disponible en: http://www.planeducativonacional.unam.mx

35. Dilnot J, Hamilton L, Maughan B, Snowling MJ. Child and environmental risk factors predicting readiness for learning in children at high risk of dyslexia. Dev Psychopathol. 2017;29:235-44.

36. Kornilov SA, Lebedeva TV, Zhukova MA, Prikhoda NA, Korotaeva IV, Koposov RA, et al. Language development in rural and urban Russian-speaking children with and without developmental language disorder. Learn Individ Differ. 2016;46:45-53.

37. Agarwal D, Chaudhary SS, Sachdeva S, Misra SK, Agarwal P. Prevalence of developmental delay and factors affecting the development status among under 5 children in an urban slum of Agra City. Natl $J$ Community Med. 2018;9:474-9.

38. Valla L, Wentzel-Larsen T, Hofoss D, Slinning K. Prevalence of suspected developmental delays in early infancy: results from a regional population-based longitudinal study. BMC Pediatr. 2015;15:215

39. Korpilahti $P$, Kaljonen A, Jansson-Verkasalo E. Identification of biological and environmental risk factors for language delay: the Let's Talk STEPS study. IInfant Behav Dev. 2016:42:27-35.

40. Law J, Garrett Z, Nye C. Speech and language therapy interventions for children with primary speech and language delay or disorder. Cochrane Database Syst Rev. 2005;(1):1-85.

41. Okuda PMM, Pinheiro FH. Motor performance of students with learning difficulties. Procedia Soc Behav Sci. 2015;174:330-8.

42. Conti-Ramsden G, Durkin K, Toseeb U, Botting N, Pickles A. Education and employment outcomes of young adults with a history of developmental language disorder. Int J Lang Commun Disord. 2018;53:237-55.

43. Winstanley M, Webb RT, Conti-Ramsden G. More or less likely to offend? Young adults with a history of identified developmental language disorders. Int J Lang Commun Disord. 2018;53:256-70.

44. Baixauli-Fortea I, Roselló-Miranda B, Colomer-Diago C. Relaciones entre trastornos del lenguaje y competencia socioemocional. Rev Neurol. 2015;60(Supl 1):S51-6.

45. Norbury CF, Vamvakas G, Gooch D, Baird G, Charman T, Simonoff E, et al. Language growth in children with heterogeneous language disorders: a population study. J Child Psychol Psychiatry. 2017:58:1092-105. 